\title{
Lidil
}

Revue de linguistique et de didactique des langues

$44 \mid 2011$

Langues, minor(is)ations et marginalisations

\section{Présentation de soi et projection en contexte homoparental : la construction langagière d'un projet de famille}

\section{Luca Greco}

\section{(2) OpenEdition \\ Journals}

Édition électronique

URL : http://journals.openedition.org/lidil/3137

DOI : 10.4000/lidil.3137

ISSN : 1960-6052

Éditeur

UGA Éditions/Université Grenoble Alpes

\section{Édition imprimée}

Date de publication : 15 décembre 2011

Pagination : 43-62

ISBN : 978-2-84310-212-7

ISSN : $1146-6480$

Référence électronique

Luca Greco, "Présentation de soi et projection en contexte homoparental : la construction langagière d'un projet de famille », Lidil [En ligne], 44 | 2011, mis en ligne le 15 juin 2013, consulté le 03 mai 2019. URL : http://journals.openedition.org/lidil/3137; DOI : 10.4000/lidil.3137 


\title{
Présentation de soi et projection en contexte homoparental : la construction langagière d'un projet de famille
}

\author{
Luca Greco*
}

\begin{abstract}
RÉSUMÉ
À partir d'une ethnographie multi-sites menée dans une association de parents et de futurs parents gay et lesbiens, j'analyserai les modalités discursives de présentation de soi. Mon attention se focalisera sur la façon dont les participants, tout en imaginant au futur une présentation de soi en tant que père ou mère de $X$, mettent en scène un réseau intersubjectif au sein duquel des dialogues entre les parents, les enfants et les proches ont lieu. Ce point me permettra de souligner la dimension réflexive entre désir d'enfant et pratiques langagières et de conclure avec un retour réflexif sur mon statut d'observateur participant au sein de l'enquête.
\end{abstract}

\begin{abstract}
This article is based on fieldwork I conducted among members of a French association of current and future gay and lesbian parents. It investigates how participants construct their desire to become parents. More specifically, I focus on the role of imagination and future parents' ability to project new events in their narratives and interactions with other participants. This study sheds light on sequential, categorial and intersubjective dimensions that shape participants' identity construction and on the reflexive relationship between language planning and family construction.
\end{abstract}

Au cours de nos vies, nous nous présentons sans cesse aux autres avec une variété incroyable de moyens et dans une gamme infinie de situations. Que ce soit au travail, dans la vie de tous les jours, en famille ou avec les amis, nous accomplissons nos identités dans et par cet outil puissant, créateur de réalités qu'est la présentation de soi. Pensons par

* ILPGA - Paris 3-Sorbonne Nouvelle. E.A. 1483 RFC. 
exemple aux pratiques de coming out et aux façons dont nous dévoilons notre identité sexuelle aux autres. Quels mots, quelles tournures utiliser? Et encore, faut-il le dire à tout le monde? Au cercle intime de la famille? Aux collègues de travail? Et surtout, quand? Attendre qu'on nous pose la question? Profiter d'un moment propice pour les confidences ${ }^{1}$ ? $\mathrm{Ou}$ l'annoncer d'une façon plus ou moins abrupte? Des problèmes bien connus par la communauté LGBTQ ${ }^{2}$ mais aussi des questions cruciales pour une étude sociolinguistique, interactionnelle des pratiques identitaires. Dans ce cadre, l'identité s'accomplit dans et par le langage qui devient l'outil et l'espace au sein desquels nous construisons nos vies, nous nous positionnons dans le monde, nous agissons avec les autres en mobilisant des dispositifs catégoriels et les formes linguistiques les plus appropriées pour le contexte en cours.

Selon Judith Butler (2005 : 51) la présentation de soi contient deux caractéristiques principales : d'une part, elle constitue un effort pour communiquer de l'information sur soi; d'autre part, elle recrée et met à nouveau en place les présomptions tacites de la communication et des relations qui structurent la manière de nous constituer en tant que sujets dans le monde. Ainsi, la présentation de soi recrée les conditions mêmes de l'interlocution et du rapport à l'autre tout en s'inscrivant dans une histoire qui nous dépasse mais qui rend possible ce que nous faisons avec le langage. De ce fait, nous apprenons tout le temps à construire des présentations qui soient les plus pertinentes pour la situation et les interlocuteurs avec lesquels nous interagissons. Les mots que nous choisissons et qui vont donner de la chair à nos présentations ne nous appartiennent pas complètement, ils nous échappent en quelque sorte. Combien de fois avons-nous éprouvé un sentiment d'inadéquation par rapport aux catégories (femme, homme, sociolinguiste, mère, père, fille, fils...) censées nous constituer en tant que sujets ? Cependant, c'est dans cette tension que nous réussissons à accomplir nos identités tout en jouant, en rejouant ou en déjouant le répertoire des catégories transmises dans nos cultures et dans nos sociétés (Butler, 2005).

Goffman qui a été un des sociologues les plus influents du siècle passé dans le domaine des interactions sociales définit la présentation de

1. Pour une étude sur la relation parfois problématique entre confidence et présentation (ou dévoilement) de soi, voir C. Kerbrat-Orecchioni et V. Traverso (éds) (2007), Confidence / dévoilement de soi dans l'interaction, Tubingue, Niemeyer.

2. Lesbian, Gay, Bisexual, Transexual, Queer. 
soi en jouant sur un rapport dialectique entre l' « expression » que nous véhiculons avec nos présentations et l'«impression » que les autres en retirent :

[...] j'examinerai ici de quelle façon une personne, dans les situations les plus banales, se présente elle-même et présente son activité aux autres, par quels moyens elle oriente et gouverne l'impression qu'elle produit sur eux, et quelles sortes de choses elle peut ou ne peut pas se permettre au cours de sa représentation. (Goffman, 1973 : 9)

Ainsi, la présentation de soi est une pratique interactionnelle : elle s'adresse aux autres et elle est pensée pour un auditoire et un contexte précis. Dans ce cadre, des auteurs s'inscrivant dans l'analyse de discours y ont vu un trait constitutif de toute interaction sociale par le fait que toute prise de parole implique toujours une présentation de soi, autrement dit, un ethos (Amossy, 2010 : 7). D'autres chercheurs ont souligné plutôt la dimension thématique (Traverso, 1998 : 194) ou plus strictement interactionnelle en focalisant leur attention moins sur les thèmes introduits par le locuteur que par la façon dont l'interlocuteur va reconnaitre dans le compte-rendu effectué par le locuteur un format reconnaissable en tant que «présentation de soi » (Antaki et al., 2005).

Dans cette perspective, la présentation de soi est identifiable par des patterns interactionnels et peut être définie comme un ensemble de méthodes mobilisées par les participants pour se présenter aux autres en mobilisant des ressources linguistiques, des dispositifs catégoriels, des formats interactionnels pertinents pour la situation en cours. Voici, dans l'extrait ci-dessous, un exemple tiré d'un tour de table précédant une conférence d'un spécialiste sur la parenté et organisé par une association parisienne de parents et futurs parents gays et lesbiens ${ }^{3}$ :

\section{TDTGOD 254-261}

1 Va voilà

$2 \quad(3)$

3 JY donc jean-yves/ je suis le parent social d'un petit

4 garçon de deux ans et demi/ qui est né en maternité

5 pour autrui... avec Hubert avec qui je vis depuis

6 seize ans/ $\mathrm{xxx}$ depuis très longtemps pour ne pas

$7 \quad$ dire trop longtemps

8 Va ((rire))

3. Pour les conventions de transcription, se reporter à l'annexe en fin d'article. 

tout.

Dans cet exemple, la présentation de soi de JY (1.3-7) est encadrée par la prise de parole (1. 1) et le rire de VA (1. 8), l'animatrice du tour de table. JY prend le tour après un lieu de transition de parole possible (voir la pause 1.2) et en suivant l'ordre des participants disposés autour de la table. Le « voilà » de l'animatrice (1. 1) est appréhendé comme une ressource clôturant la présentation précédente et ouvrant le champ à une nouvelle, la sienne. La présentation de JY (1.3-7) mobilise plusieurs dispositifs catégoriels : le nom propre, le statut parental et de couple. Ces dispositifs sont construits dans le temps pendant lequel il garde la parole malgré de nombreux points de transition de parole possibles (voir l'intonation montante, la micro-pause 1.5) ${ }^{4}$. Après le rire de l'animatrice (1. 8), la participante assise juste à côté de lui prend la parole (1.9) et de fait reconnait la présentation de JY comme étant close. Ce court extrait et l'analyse que nous avons proposée nous permettent de dresser une sorte de bilan autour des dimensions que l'on peut étudier dans une approche multidimensionnelle de la présentation de soi :

a) la dimension langagière : quelles ressources verbales (et non verbales) mobilise-t-on pour se présenter à son auditoire?

b) la dimension catégorielle : quels dispositifs identitaires choisiton pour se présenter d'une façon adéquate par rapport à la situation et aux interlocuteurs?

c) la dimension séquentielle : dans quel environnement interactionnel la présentation de soi est-elle censée émerger?

d) la dimension temporelle : de quelle façon la présentation des participants change-t-elle au cours de l'enquête et de leur vie? Et enfin,

e) la dimension réflexive : comment les présentations des participants, qu'elles soient au présent ou projetées dans un futur hypothétique (« quand je serai $X /$ si je deviens $X » . .$. ), contribuent-elles à la construction d'un désir et d'un projet de vie?

En prolongeant les travaux que nous avons consacrés à une approche multidimensionnelle de la présentation de soi (Greco, 2010, 2006, sous presse, à paraitre), nous nous concentrerons dans ce texte sur la dimension

4. Pour une analyse systématique de ce point, nous renvoyons à Greco, 2006. 
réflexive de la présentation de soi tout en faisant référence à la dimension catégorielle et séquentielle. Le cadre théorique des analyses présentées dans ce texte renvoie à une conception praxéologique du langage conçu en tant que mode d'action, collectivement accompli et historiquement ancré. Cette vision puise ses racines au sein de l'anthropologie linguistique (Duranti, 1997) mobilisant les approches interactionnelles d'inspiration conversationnelle (Sacks, 1992; Sacks, Schegloff et Jefferson, 1974) et les méthodologies ethnographiques. Les analyses présentées dans ce texte souligneront ainsi le placement séquentiel de la présentation de soi ainsi que sa dimension catégorielle à partir d'un objet dont les coordonnées théoriques et analytiques croisent tant l'ethnométhodologie que l'anthropologie linguistique et les études de genre.

\section{Le terrain et les données}

Les données présentées dans cet article sont issues d'une recherche de terrain que j'ai menée dans une association de parents et de futurs parents gays et lesbiens en région parisienne. Pendant la durée de ce terrain (trois ans), j'ai eu la possibilité d'observer, de participer à et d'enregistrer un bon nombre d'activités proposées par l'association : des groupes de parole, des échanges sur la mailing list de l'association, des rencontres organisées par l'association et adressées à ses membres, des cafés politiques, des manifestations et des entretiens avec les adhérents. De ce fait, mon dispositif ethnographique s'est assez vite caractérisé par une multitude d'activités proposées par l'association ayant lieu dans des espaces divers et variés. Le choix de travailler sur la présentation de soi et sur ses multiples dimensions m'a amené à mettre en place un dispositif ethnograhique multi-sites (Marcus, 1995) me permettant de suivre les activités des membres de l'association dans plusieurs endroits différents en jonglant à la fois avec l'identité de « participant » et d' " observateur » selon les moments, les contextes et les interlocuteurs rencontrés au fil de mon enquête (Greco, 2010) ${ }^{5}$.

Lors du terrain, j'ai été impressionné par le rôle que jouait le langage dans la construction d'un projet parental. La construction d'une famille s'imbriquait ainsi avec une sorte de « planification linguistique » autour

5. Il serait intéressant de se demander dans quelle mesure une ethnographie menée dans un «terrain sensible » nous oblige à reconfigurer, à fragmenter et à problématiser la spatialité de l'enquête. 
des termes de parenté choisis pour désigner et nommer les parents biologiques et sociaux. De ce fait, un certain nombre de questions étaient débattues lors des rencontres : comment appeler la compagne ou le compagnon du parent biologique? Maman, papa? Ou bien, fallait-il trouver un autre terme plus adéquat pour signifier la position de parent social ( $v s$ biologique) vis-à-vis de l'enfant ? Et encore, comment l'enfant allait-il se débrouiller avec ses pairs en parlant de sa famille? J'ai deux mamans? ou j'ai une mère et une marraine? Souvent ces problèmes faisaient l'objet d'importantes discussions lors des groupes de parole, des échanges sur le net ou des réunions entre membres de l'association. Souvent, dans le cadre de ces discussions, les participants avaient aussi la tendance à se projeter dans le futur en imaginant des discussions soit entre parents et enfant, soit entre enfants eu égard à leurs configurations parentales. C'est autour de cet aspect que je voudrais recentrer les analyses contenues dans ce texte. Après avoir présenté un cas exemplaire de présentation de soi projetée au futur (§3), je me pencherai sur d'autres extraits en me focalisant sur la temporalité et l'intertextualité des évènements rapportés ainsi que sur la dimension morale de ces présentations $(\S 4)$. Enfin, je terminerai avec un retour réflexif sur ma propre présentation au cours de l'enquête $(\S 5)$, ce qui me permettra par la suite de dresser un bilan conclusif $(\S 6)$ sur les questions débattues dans ce texte.

Les données utilisées dans cet article sont issues d'un corpus assez hétéroclite composé d'interactions au sein d'un groupe de parole, d'un tour de table organisés par les membres de l'association et d'échanges ayant lieu dans la mailing list de l'association. Les analyses présentées dans ce texte ne prétendent ni dresser des généralisations sur la dimension réflexive de la présentation de soi ni proposer un modèle qui rendrait compte des contraintes contextuelles sur la configuration linguistique et interactionnelle de la présentation de soi. Dans ce cadre, l'objectif que je poursuis est double. D'une part, je souhaite poser des jalons pour l'étude de la dimension réflexive de présentation de soi. D'autre part, je contribue avec ce travail à la mise en place d'une approche multidimensionnelle des pratiques identitaires.

\section{Un cas exemplaire}

Dans cet extrait, Hilary, l'animatrice d'un groupe de parole thématique « futurs parents », imagine la façon dont l'enfant d'un couple homoparental pourra parler de sa famille au futur : 


\section{GDP34-78jeux-billes}

\begin{tabular}{|c|c|c|}
\hline 1 & $\mathrm{H}$ & il y jouera avec son discours i- se trouve un discours \\
\hline 2 & & l'enfant mais ça nous on maitrisera pas forcément. \\
\hline 3 & $\mathrm{I}$ & hum hum \\
\hline 4 & $\mathrm{H}$ & on lui donnera des billes pour s'exprimer sur nous la \\
\hline 5 & & façon: ${ }^{\circ}(\text { donc }, \text { dont })^{\circ}$ comment on va lui dire mais \\
\hline 6 & & [après] \\
\hline 7 & $\mathrm{E}$ & [hum] \\
\hline 8 & $\mathrm{H}$ & lui/ pou- pou- parl- rapport à ses copains i- \\
\hline 9 & & s'débrouillera hein: [i- ] fera i- \& \\
\hline 10 & $\mathrm{E}$ & [hum] \\
\hline 11 & $\mathrm{H}$ & \& f'ra/ i- f'ra:/ \\
\hline 12 & $\mathrm{I}$ & i- f'ra avec \\
\hline 13 & $\mathrm{H}$ & [i- f'ra avec \\
\hline \multicolumn{3}{|c|}{ ((plusieurs tours omis)) } \\
\hline 23 & $\mathrm{H}$ & [parce que/ l'enfant i- va s'dire/] \\
\hline 24 & & j'ai pas d'père mais alors j'ai deux mamans/ ou: j'ai \\
\hline 25 & & une marraine/ \\
\hline 26 & & [ah bon ] t'as une marraine/ euh: ben moi aussi j'ai \\
\hline 27 & $\mathrm{E}$ & {$\left[{ }^{\circ}\right.$ ouais hum $\left.{ }^{\circ}\right]$} \\
\hline 28 & $\mathrm{H}$ & une marraine euh: ah ouais mais moi j'ai une marraine \\
\hline
\end{tabular}

La dimension indexicale des interactions imaginées entre l'enfant et son environnement social sont soulignées à plusieurs reprises par Hilary (« il jouera avec son discours »1. 1, « il se débrouillera »1. 9, « il fera avec $\gg 1.9-13$ ) et ratifiées aussi par les autres participants. Ensuite, l'animatrice reprend la parole et met en scène un dialogue entre l'enfant et ses pairs (1. 23-28). Ce dialogue est préfacé par la ressource «parce que » (1.23) ayant une portée aussi bien rétrospective que prospective (« i- va s'dire »1.23). De ce fait, ce qui va suivre sera présenté comme une sorte d'illustration imagée de l'hypothèse énoncée plus haut. Ainsi, la façon dont on imagine l'enfant interagir avec ses pairs à propos de sa configuration familiale est à la fois présentée sous l'angle du jeu (23-28) et dans sa dimension improvisationnelle (1.1-13). À ce propos, Hilary « anime» (Goffman, 1981) les voix des enfants en interaction sous le mode d'un jeu dans lequel les participants prennent la parole dans une sorte de défi touchant à leurs configurations parentales : «j'ai pas de père mais j'ai deux mamans ou une marraine », « ah t'as une marraine ben moi aussi j'ai une 
marraine » (1.24-28) ${ }^{6}$. Dans cet extrait, le choix du terme de parenté pour désigner le parent social est situé dans un réseau intersubjectif de relations entre l'enfant et ses pairs. C'est dans ce sens que l'intersubjectivité en tant que prise en compte de l'autre, avant même que cette rencontre puisse avoir lieu (Duranti, $2010: 9^{7}$ ), acquiert ici tout son sens et c'est pourquoi je me tournerai vers l'analyse d'un ensemble d'extraits illustrant le phénomène de la projection au futur dans la présentation de soi.

\section{La projection dans le futur}

La présentation de soi est toujours ancrée dans un passé qui la rend possible tout en étant orientée vers un présent et un futur envisageables. Ce point a été souligné à plusieurs reprises par plusieurs chercheurs qui ont théorisé la relation entre pratiques sociales et contexte. Dans ce cadre, Bourdieu (1980 : 89) offre avec la notion d'habitus une théorie des pratiques conçues en termes de tension agentive entre un dépôt d'expériences passées et les dispositions à l'action pour (et dans) le futur. Ainsi, les pratiques, tout en étant situées dans un contexte qui les précède, sont constitutives d'autres contextes possibles et imaginables (Heritage, 1984; Ochs, 1992). Cet aspect a été souligné par deux anthropologues du langage ayant travaillé sur les narrations entre pairs ou en famille. Ainsi, Elinor Ochs (1994), en s'inspirant d'Heidegger et de Ricœur, a montré clairement comment les narrations ont la capacité de faire face au passé et de se projeter vers le futur en rendant ainsi nos vies cohérentes. M. H. Goodwin (1990), de son côté, a également montré comment les récits dits d' "instigation » entre gamines projetaient dans le futur des réactions vis-à-vis de la partie offensante (1990 : 277). C'est dans

6. Ce type de performance verbale est « proche ", à mon avis, des pratiques de vannes et d'instigation observées et analysées par Labov (1972) et M. H. Goodwin (1990). Une analyse approfondie permettrait de trouver les indices nous permettant d'argumenter cette hypothèse.

7. Depuis plusieurs années, Duranti est vivement engagé dans un travail passionnant sur l'intersubjectivité et sur une relecture anthropologique de cette notion qui puise ses racines dans la philosophie d'Husserl et de Levinas mais aussi dans la sociologie de Schütz. Pour une présentation de cette notion et de sa pertinence pour une approche interactionnelle (Duranti, 2006, 2009, 2010). Qu'il soit ici remercié pour avoir partagé avec moi des intenses discussions sur l'agentivité et sur l'intersubjectivité dans l'interaction lors de plusieurs séances de travail. 
la capacité de faire l'expérience des autres en tant que co-participants de nos interactions passées et futures que l'intersubjectivité se construit (Schütz, 1932 : 9). Dans ce cadre, la prise en compte de l'autre peut avoir un effet configurant sur l'accomplissement des pratiques et sur le choix d'un dispositif catégoriel comme dans l'extrait suivant :

\section{Faire-partMailinglist}

Bonjour,

Notre petit bonhomme pousse bien et le grand rendez-vous approche... Dans deux mois, nous serons une famille! Il est temps de penser aux faire-parts!! Nous voudrions quelque chose d'assez clair sur notre situation particulière (2 mamans, IAD) notamment pour la famille et les amis. En même temps, nous allons devoir annoncer la nouvelle à des relations de travail avec lesquelles nous n'avons pas forcément envie de rentrer dans les détails... Devons-nous faire deux versions?

La prise en compte de plusieurs types d'interlocuteurs (famille et amis, relations de travail) configure la façon dont le couple de femmes se présentera aux autres tout en montrant un lien entre catégories et pratiques. Dans l'extrait suivant, Hilary pose des questions à un couple de femmes sur la façon dont elles parleront du donneur à l'enfant :

\section{GDP-8-30-donneurc'estpasunpère}

\begin{tabular}{|c|c|}
\hline $\mathrm{H}$ & [est ce que vous allez parler du donneur/ \\
\hline I & bon il nous a parlé de pèrel je lui ai dit non il \\
\hline & aura pas de pè:re dans un sens/ langage/ langagier/ il \\
\hline & aura deux mè:res/ (1) par contre il aura pas un pè:re/ \\
\hline & par contre il aura des o:ncles des parents/ .. euh des \\
\hline & parrains/ qui remplaceront/= \\
\hline $\mathrm{H}$ & =est-ce que le donneur le donneur n'est pas un père/ \\
\hline & .. \\
\hline $\mathrm{N}$ & [non] \\
\hline I & [no:n/] le donneur en fait c'est quelqu'un qui nous a \\
\hline & $\begin{array}{l}\text { donné l'autorisation qui a donné la graine pour qu'on } \\
\text { puisse construire e- un couple eh une famillel. }\end{array}$ \\
\hline
\end{tabular}

Les réponses d'Isa et de Nathalie s'insèrent au sein de deux paires adjacentes question-réponse (1. 1-6 et 1. 7-12). Dans la première paire, suite à une question fermée sur le donneur (1. 1), Isa répond d'une façon quelque peu complexe. Au lieu de répondre par une réponse attendue d'un point de vue morphosyntaxique (oui/non), elle réplique en mettant 
en scène une conversation que les deux femmes ont eue avec un psychologue en Belgique (1.2). Dans cette scène, elle reproduit une réponse au discours rapporté (1.2-3) suite au thème du «père » initié par le médecin (1.2). Au cours de cette réponse, nous assistons à un jeu catégoriel assez intéressant. L'impossibilité langagière d'un père (1. 2-3 « il aura pas de pè:re dans un sens/ langage/ langagier/») est justifiée avec la présence de « deux mères » et la présence d'un environnement social masculin qui remplaceront la figure du père (1. 4-6). Ensuite, Hilary reprend la parole pour poser une question sur l'équivalence ontologique possible entre « donneur » et «père » (1. 7). Suite à une micro pause (1. 8) les deux femmes se sélectionnent d'une façon simultanée (1. 9-10). Alors que Nathalie répond avec une réponse attendue $(1.9$ « non ») suite à une question fermée, Isa développe sa réponse (1. 10-12) en mobilisant une narration autour du donneur et de la «graine », que j' ai entendue maintes fois lors de ma participation aux activités proposées par l'association, et qui est censée expliquer la figure du donneur auprès des enfants. Ce qui est en jeu dans les procédés de dénomination et de catégorisation du donneur est moins la présentation de soi que la présentation d'un autre absent. Cependant, la présentation du donneur aura des effets sur la présentation de soi de l'enfant. C'est la façon dont les parents ou les géniteurs sont nommés en tant que « père », «mère » etc. qui va donner une place à l'enfant dans le réseau de sa sociabilité. C'est enfin dans ce réseau à la fois parental et nominal que l'enfant pourra se présenter aussi bien en tant que « je suis fils de » mais aussi par inférence grâce au syntagme «mon père », « mes mères », « mon parrain », « mes oncles » ${ }^{8}$. Dans l'extrait suivant, le groupe de parole est à nouveau focalisé sur la dénomination du donneur (1.1-3) :

\section{GDP-58-74-il va dire}

$1 \quad \mathrm{H} \quad$ mais ce qu'il y a c'est que quand on dit ton père ton

2

3

4 E $\quad \mathrm{mhm}$

5 I je lui dirai qu'il a un papa biologique comme tous

6

7 les enfants/ qu'il est normal/ il a un papa biologique/

8. Ainsi, c'est la nature relationnelle des termes de parenté qui fait qu'un terme de parenté comme «père » puisse donner des informations sur la position d'un locuteur au sein d'un réseau parental au moment où il dit « mon père ». 


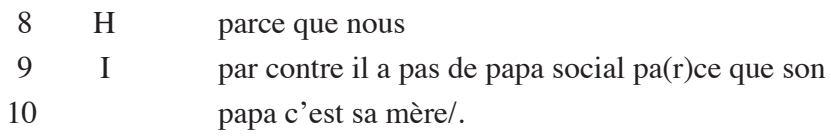

À ce propos, plusieurs choix sont proposés par Hilary : « ton père », «le donneur », « le géniteur » (1.2-3) et insérés dans un cadre dialogique avec l'enfant («tu peux lui dire»1.2, «tu vas lui dire »1.3). Suite aux propositions d'Hilary, Isa formule sa réponse en deux parties. D'une part (1. 5-7), elle construit sa réponse sous forme de discours rapporté avec l'enfant ( « je lui dirai » 1.5) en mobilisant la catégorie ad hoc «papa biologique » suivie d'un account ${ }^{9}$ moral au présent (« il est normal» 1.6). D'autre part, elle reformule sous un angle inédit les paires standardisées catégorielles (Sacks, 1972) père/papa - mère/maman (1.9-10). Dans ce cadre, la présence d'un donneur catégorisé ici comme un "papa biologique » rend impossible l'appellation du donneur en tant que «papa » et sa catégorisation en tant que «papa social » étant cette fonction remplie par la mère (1.9-10). Il est intéressant aussi de remarquer que la reconfiguration des dispositifs parentaux (1.9-10) comme la catégorisation ad hoc («papa biologique») et l'account précédents sur la normalité se font dans le temps de la conversation au présent et échappent à la projection au futur. Ainsi, d'un point de vue séquentiel et temporel, on peut assister à l'émergence d'un pattern qui prendrait les formes suivantes :

1. Mise en contexte de la situation avec éventuellement une question (1. 1-3);

2. Réponse au futur au discours rapporté (1. 5-6) ;

3. Account au présent (1. 6-7, 1. 9-10).

Ce modèle se répète plus loin dans la même conversation où un autre participant (E) en écho à la question posée par Hilary (1. 1-3) prend la parole pour exprimer son avis :

2. Réponse au futur avec discours rapporté :

\section{5bis. GDP-232-273-donneur-géniteur}

17 E le gamin quand il s'ra à l'école quand j'lui dirai alors

18 alors bon puisque [c'est vous on parle de vous] euh: où

9. Par le terme account nous entendons surtout des activités d'explication et de justification se situant après la description d'un fait. Pour cette notion, nous faisons bien entendu référence au travail pionnier de Scott et Lyman (1968) et à celui de Goffman (1974) sur les stratégies de réparation. 


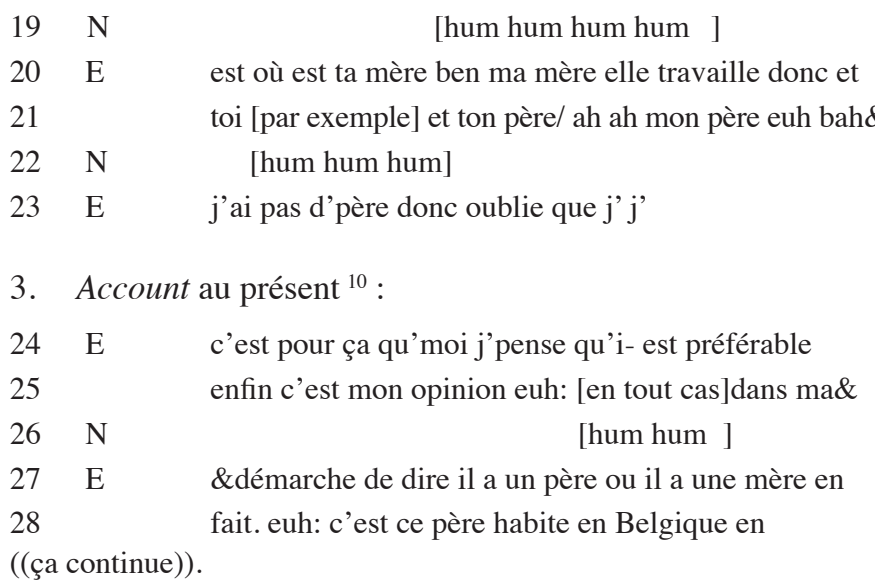

Un trait commun à ces extraits est aussi la façon dont le futur est temporellement imaginé et construit dans les récits des participantes. Si l'on s'attarde à nouveau sur quelques fragments des extraits analysés, on se rend compte que la façon dont les participantes projettent au futur une configuration parentale avec l'enfant (extrait 4), ou des conversations avec les enfants (extrait 5) ou encore en animant la voix de l'enfant avec ses pairs (extrait 5bis) prend les allures d'un évènement accompli, presque passé. C'est en effet, une des caractéristiques que Schütz avait remarquée à propos de ce qu'il appelle le futur past tense. En s'appuyant sur une des figures mythologiques grecques Tiresias et sur la façon dont on produisait des prédictions au futur comme si elles avaient déjà eu lieu (Schütz, 1964 : 278-279), Schütz théorise une modalité pratique de construction au futur au carrefour entre la prophétie et le travail historiographique. Le travail temporel accompli par les participantes est de ce point de vue tout à fait intéressant car tout en projetant une action au futur, elles présentent l'action comme si elle avait déjà eu lieu une fois qu'elle est rapportée à d'autres interlocuteurs dans le temps de la conversation. C'est dans cette imbrication entre passé, futur et présent

10. Ce serait intéressant d'étendre l'analyse à d'autres cas semblables. Elizabeth Stokoe (2010) a trouvé dans son corpus d'interviews à la radio anglaise des structures assez similaires au sein du tour d'un participant : description, catégorisation et connaissances de sens commun (common knowledge component (2010 : 431)). 
que nos interactions ont lieu et que des liens se construisent entre les participantes dans un cadre irréductiblement intersubjectif ${ }^{11}$.

\section{Un retour réflexif}

Toute expérience ethnographique ne peut faire l'économie d'une présentation de soi du chercheur mobilisant devant ses interlocuteurs des dispositifs identitaires relevant de l' " intime » et du «personnel». Il y a tout un courant de l'anthropologie féministe, gay, lesbienne, queer (Lewin et Leap, 1996, 2009; Lewin, 2005) qui n’a cessé depuis des années de s'interroger sur la façon dont les identités du chercheur (femme, noire, lesbienne, trans, gay...) configurent les relations avec les personnes rencontrées sur le terrain ainsi que les objets d'étude mêmes. Lors de mon terrain à l'APGL, j'ai été plusieurs fois confronté à mon identité d'homme, de gay, de père, lors des interactions avec les participants. Lors de mon arrivée au sein du groupe de parole, une des conditions qui m'ont été posées par l'animatrice était celle de me questionner sur mon identité de père potentiel et de m'engager ainsi avec les autres participants dans une discussion le plus possible «symétrique ». Au fur et à mesure que ma participation au sein du groupe se faisait importante ${ }^{12}$, il devenait de plus en plus difficile de contrôler les frontières entre «observation » et «participation », « personnel » et « scientifique ». Jusqu'au jour où j' ai reçu un courriel dans lequel on me proposait de devenir père d'un enfant et de m'engager ainsi dans un projet de co-parentalité avec un couple de femmes. Suite à cette requête pour le moins inattendue, j'ai répondu en leur disant que je n'étais pas encore prêt et que mon projet n'était pas encore défini. Suite à cet évènement, j'en avais parlé aux autres membres du groupe jusqu'à ce que cela devienne un topic du groupe de parole grâce à une question de l'animatrice (1. 1-2) :

\section{GDP-termoimême65-120}

$1 \mathrm{H}$ et toi ça t'a interpellé sur (2) - fin $^{\circ}$-fin ${ }^{\circ}$

2 parentalité potentielle/

3

11. Ce point a été montré par Lisa Capps et Elinor Ochs (1995: 62) dans l'étude des narrations d'une femme agoraphobe.

12. Le lecteur pourra se référer à Greco (2010) pour une analyse approfondie de mon parcours ethnographique au sein de l'association. 


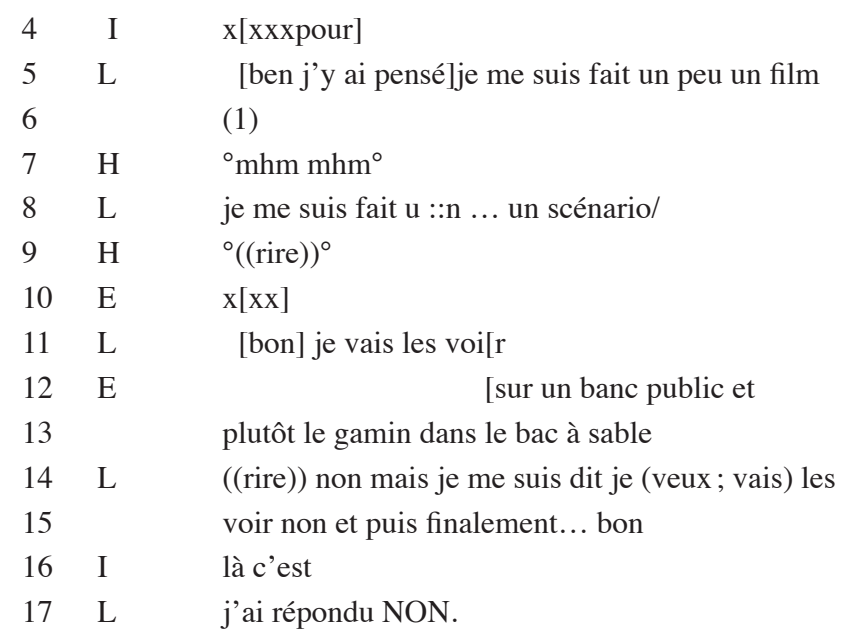

Dans cet extrait, suite à la question d'Hilary (1. 1-2), j'enchaine avec une narration (1.5-11) au sein de laquelle après avoir répondu à la question de l'animatrice ( «ben j'y ai pensé » 1. 5), j'imagine le début d'une scène au futur proche (« je vais les voir» 1.11 ) dont les éléments seront introduits grâce à l'intervention d'un autre participant (1. 12-13) au moyen d'un procédé de co-construction syntaxique. Ensuite, je reprends la parole (1.14) pour répondre à la fois à la question initiale de l'animatrice (1. 14) et au mail que j'avais reçu par les deux femmes (1. 17). La catégorisation dans ce cas n'est pas explicite comme dans les extraits précédents. Elle est inférée à la fois par la question d'Hilary (1. 1-2) et par ma réponse. Ce que l'on peut inférer au niveau catégoriel, c'est à la fois le dispositif de «père » (ou plutôt de "pas père ») mais aussi celui de «participant à l'interaction» au même titre que les autres. Ce que l'on peut relever est un paradoxe catégoriel : alors que ma participation dans le groupe se fait de plus en plus intense grâce à des prises de parole importantes et à des liens de plus en plus forts avec les autres membres du groupe, mon questionnement sur une paternité éventuelle arrive à son terme avec un déplacement identitaire qui me situe définitivement en dehors de la catégorie « père » (1. 17).

\section{Quelques conclusions}

D'un pont de vue théorique, les analyses présentées dans ce texte offrent des pistes intéressantes aussi bien pour l'étude des pratiques identitaires 
en contexte que pour l'analyse de la dimension temporelle et réflexive de la présentation de soi. Dans ce cadre, cet article apporte une dimension analytique et empirique aux débats théoriques émergeant au sein des études de genre et identifiant dans le langage un outil de construction identitaire important. De plus, grâce à l'analyse de l'intersection entre dimension interactionnelle, catégorielle et temporelle (voire réflexive) de la présentation de soi, cet article se propose comme un espace de dialogue entre approches séquentielles, catégorielles et linguistiques.

D'un point de vue méthodologique, la prise en compte des trajectoires identitaires accomplies par le chercheur au sein de la communauté d'accueil permet de passer de l'observation participante à l'observation de sa participation (Tedlock, 1991; Greco, 2010) en l'intégrant ainsi dans l'analyse et en en faisant un objet d'étude.

D'un point de vue strictement analytique, les analyses proposées dans cet article permettent de dresser un bilan provisoire autour de trois aspects dans la présentation de soi en contexte homoparental :

a) l'aspect réflexif : la nomination et la catégorisation des liens parentaux deviennent constitutives de la façon dont les liens sociaux sont conçus et sont mis en scène par les participants en interaction. Ainsi, un lien de parenté futur ne peut être pensé et imaginé sans une réflexion et une négociation autour des termes de parenté choisis pour désigner et nommer les parents biologiques et sociaux;

b) l'aspect intersubjectif : la présentation de soi passe par un réseau d'intersubjectivité assez complexe dans lequel on rend compte de la place du parent social ou du donneur par rapport à l'enfant et aux personnes s'occupant de son éducation. Dans ce cadre, ce qui est souligné par les participants c'est la prise en compte d'interactions à venir, projetées dans un futur, plus ou moins proche, désiré. La façon dont les interactants se projettent dans le futur nous éclaire aussi sur l'ancrage temporel des actions et sur les modes de constitution de l'intersubjectivité;

c) l'aspect séquentiel : l'analyse séquentielle et catégorielle de la dimension réflexive de la présentation de soi permet de montrer comment la mise en scène aussi bien des dialogues avec l'enfant que des interactions dans lesquelles on anime la voix de l'enfant en train d'échanger avec d'autres participants s'insère dans un réseau séquentiel et temporel : mise en contexte de la situation avec éventuellement une question-réponse au futur au discours rapporté -account au présent. 


\section{RÉFÉRENCES BIBLIOGRAPHIQUES}

Amossy R. (2010) : La présentation de soi. Ethos et identité verbale, Paris, PUF.

AntAKi C., BARnes C. et LeudAr I. (2005) : « Self-disclosure as a situated interactional practice », British Journal of Social Psychology, $\mathrm{n}^{\circ} 44$, p. 181-199.

Bourdieu P. (1980) : Le sens pratique, Paris, Minuit.

Butler J. (2005) : Giving an Account of Oneself, New York, Fordham University Press, trad. française 2007.

Capps L. et Ochs E. (1995) : Constructing Panic: The discourse of agoraphobia. Harvard, Harvard University Press.

Duranti A. (1997) : Linguistic Anthropology, Cambridge, Cambridge University Press.

- (2006) : « Narrating the political self in a campaign for U.S. Congress », Language in Society, $\mathrm{n}^{\circ} 35, \mathrm{p} .467-497$.

- (2009) : «The Relevance of Husserl's Theory to Language Socialization », Journal of Linguistic Anthropology, vol. 19, Issue 2, p. 205-226.

- (2010) : «Husserl, Intersubjectivity and Anthropology », Anthropological Theory, vol 10(1), p. 1-20.

Goffman E. (1973) : The Presentation of Self in Everyday Life, trad. française La mise en scène de la vie quotidienne. La présentation de soi, vol. 1, Paris, Minuit.

- (1974) : Interactional Rituals, trad. française Les rites d'interaction, Paris, Minuit.

- (1981) : Forms of Talk, Philadelphia, University of Pennsylvania Press (trad. française Éditions Minuit « Façons de parler »).

Goodwin M. H. (1990) : He-Said-She-Said: Talk as a Social Organization among Black Children. Bloomington, Indiana University Press.

GreCo L. (2006) : «Identité, contexte et pratiques sociales : l'accomplissement interactionnel d'un tour de table », Verbum, tome XXVIII, $\mathrm{n}^{\circ} 2-3$ (La pertinence du contexte : contributions de l'ethnométhodologie et de l'analyse conversationnelle, numéro spécial édité par Lorenza Mondada) p. 153-174.

- (2010) : «Dispositifs de catégorisation et construction du lien social. L'entrée dans une association homoparentale », Genre, sexualité \& société, $\mathrm{n}^{\circ} 4$ octobre (revue en ligne).

- (2011) : «L'homoparentalité entre parenté, genre et langage : différenciation et binarité dans les procédés d'appellation et de catégorisation du lien parental », dans A. Duchêne et C. Moïse (éds), Langage, genre et sexualité. Québec, Éditions Nota Bene. 
- (à paraitre) : «Un soi pluriel : la présentation de soi dans les ateliers drag kings. Enjeux interactionnels, catégoriels et politiques », dans N. Chetcuti et L. Greco (éds), Le genre à l'épreuve des dispositifs de langage, de pouvoir et de catégorisation sociale, Paris, Presses de la Sorbonne Nouvelle.

Heritage J. M. (1984) : Garfinkel and Ethnomethodology, Cambridge, Polity Press.

Kerbrat-Orecchioni C. et Traverso V. (éds) (à paraitre) : Confidence / dévoilement de soi dans l'interaction, Tubingue, Niemeyer.

LABOv W. (1972) : Language in the Inner City: Studies in the Black English Vernacular. Philadelphia, University of Pennsylvania Press.

Lewin E. (éd.) (2005) : Feminist Antrhopology. A Reader, Malden MA, Blackwell Publisher.

LeWIN E. et LEAP W. L. (éds) (1996) : Out in the field. Reflections of lesbian and gay anthropologists. Urbana, University of Illinois Press.

- (2009) : Out in Public. Reinventing Lesbian/Gay Anthropology in a Globalizing World, Malden MA, Blackwell Publisher.

Marcus G. E. (1995) : « Ethnography in/of the world system: The emergence of multi-sited ethnography », Annual Review of Anthropology, $n^{\circ} 24$, p. 95- 117.

Ochs E. (1992) : «Indexing gender », dans A. Duranti et C. Goodwin (éds), Rethinking Context, Cambridge, Cambridge University Press, p. 335350.

- (1994) : «Stories that step into the Future », dans D. Biber et E. Finegan (éds), Sociolinguistic Perspectives on Register, Oxford, Oxford University Press, p. 106-135.

SACKS H. (1972) : «An initial investigation of the Usability of Conversation Materials for Doing Sociology », dans D. N. Sudnow (éd), Studies in Social Interaction, New York, Free Press, p. 31-74.

-(1992 [1964-72]) : Lectures on Conversation (2 vol), édité par G. Jefferson, introduction de E. A. Schegloff, Oxford, Basil Blackwell.

Sacks H., Schegloff E. et Jefferson G. (1974) : « A simplest Systematics for the Organisations of Turn-Taking for Conversation », Language, $\mathrm{n}^{\circ} 50, \mathrm{p} .735$.

Schütz A. (1932) : The Phenomenology of the Social World, London, Northwestern University Press (traduction anglaise de l'allemand en 1967).

- (1964) : Collected Papers II. Studies in Social Theory, The Hagues, Martinus Nijhoff.

Scott M. B. et Lyman S. (1968) : «Accounts », American Sociological Review, $\mathrm{n}^{\circ} 33(1)$, p. 46-62. 
Sтокое E. (2010) : «Gender, Conversation Analysis, and the Anatomy of Membership Categorization Practices », Social and Personality Psychology Compass, $\mathrm{n}^{\circ}$ 4/7, p. 428-438.

TedLock B. (1991) : «From participant Observation to the Observation of Participation: The Emergence of Narrative Ethnography », Journal of Anthropological Research, $n^{\circ} 47 / 4$, p. 69-94.

TRAVERSO V. (1998) : La conversation familière. Analyse pragmatique des inter-actions, Lyon, Presses universitaires. 


\section{ANNEXE}

\section{Conventions de transcription}

note le début du chevauchement entre deux locuteurs note la fin du chevauchement, lorsque cela a été jugé nécessaire

et $\backslash$ notent les montées et descentes intonatives note des intonations sous forme de liste et .. et ... notent des pauses petites, moyennes, longues notent des pauses plus longues, mesurées en secondes (à partir de 1 seconde)

: notent des allongements syllabiques

le soulignement note une emphase particulière sur une syllabe ou un mot les CAPITALES les signes ${ }^{\circ} \mathrm{ok}^{\circ}$ notent un volume fort de la voix notent un volume bas de la voix

$>$ on voulait s'échapper< note un rythme rapide dans la prononciation d'un fragment de parole

$=$ note un enchainement rapide entre deux tours de parole

$\&$ note la continuation du tour par le même locuteur, au-delà de l'interruption de la ligne de la transcription pour l'introduction d'un chevauchement par un autre locuteur

XXX notent un segment incompréhensible ((rire)) note la troncation d'un mot esquissé note l'aspiration du locuteur entre parenthèses sont notés les commentaires du transcripteur, ainsi que des phénomènes non transcrits

$<>$ délimitent le segment sur lequel porte la description entre (( ))

(deux jours) note une incertitude dans la transcription ? note une incertitude au niveau de l'identification du locuteur 
